\title{
Charge densities in impact parameter and transverse coordinate space
}

\author{
Harleen Dahiya*t \\ Department of Physics, Dr. B.R. Ambedkar National Institute of Technology, Jalandhar, 144011, \\ India \\ E-mail: dahiyah@nitj.ac.in

\section{Narinder Kumar} \\ Department of Physics, Dr. B.R. Ambedkar National Institute of Technology, Jalandhar, 144011, \\ India \\ E-mail: kumarnarindernitjeprotonmail.ch
}

Electromagnetic form factors obtained from the overlap of light front wave functions (LFWFs) have been used to study the transverse charge densities. The calculations have been carried out to develop a relation between the charge distribution of the quarks inside nucleon in the transverse coordinate space as well as in the impact parameter space.

XXIII International Workshop on Deep-Inelastic Scattering

27 April - May 12015

Dallas, Texas

\footnotetext{
* Speaker.

${ }^{\dagger}$ Authors would like to thank Department of Science and Technology, Government of India for financial support.
} 


\section{Introduction}

Study of form factors has been important in the field of hadron physics as they provide important information about internal structure of the nucleon [1,2,3]. Recently, experiments have measured and produced remarkably precise data for the electromagnetic form factors $[4,5,6]$. The nucleon electromagnetic form factors have been used to study the distribution of charge and magnetization densities of quarks inside the nucleon [7, 8]. To get a more clear picture of the nucleon structure, one can fully parameterize the structure of the nucleon in terms of generalized parton distributions (GPDs) $[9,10]$ which are functions of longitudinal momentum $x$ of the quark, the invariant momentum transfer $t$ and the skewness parameter $\xi$ which gives the fraction of the longitudinal momentum transfer to the nucleon. The Fourier transform of the GPDs w.r.t transverse momentum transfer has not only been used to study the nucleon structure in transverse impact parameter space $[11,12,13]$ but it also gives a tomographic picture of the distribution of quark charge densities inside the nucleon through the impact parameter dependent parton distribution function (ipdpdf) $q\left(x, b_{\perp}\right)$ for a quark of momentum fraction $x$ located at a transverse position $b_{\perp}$.

Transverse charge density defines a way to analyze electromagnetic form factors of hadrons moving at infinite momentum with transverse distance $b_{\perp}$ from the transverse center of momentum [14]. Charge densities have been discussed in impact parameter space for the polarized and transversely polarized nucleons $[8,15]$, in finite radius approximation [16], in scalar diquark model using transverse coordinate space [7] as well as using Sachs form factors [17, 18]. Transverse densities can also be useful in the study of the spatial distribution of the momentum $P^{+}$and can be related with the Fourier transforms of the gravitational form factors $A\left(q^{2}\right)$ and $B\left(q^{2}\right)$ [19].

The above discussion motivate us to study the charge and magnetization densities obtained from the helicity non flip and helicity flip form factors. One of the most successful model which finds its application for the quantities discussed above consists of spin- $\frac{1}{2}$ system as a composite of spin- $\frac{1}{2}$ fermion and spin-1 vector boson. We have generalized the framework of QED by assigning a mass $M$ to external electrons in the Compton scattering process, but a different mass $m$ to the internal electron line and a mass $\lambda$ to the internal photon line. In our case we take $\xi=0$ [12] which represents the momentum transfer exclusively in transverse direction leading to the study of ipdpdfs in transverse impact parameter space.

The purpose of the present communication is to determine the distribution of nucleon charge density in transverse coordinate space and in impact parameter space using light front wave functions (LFWFs) of simple spin- $\frac{1}{2}$ objects like dressed quark or a dressed electron in theory. Further, it would be significant to develop a relation between the distribution of quark charge densities inside nucleon in the transverse coordinate space as well as in the impact parameter space particularly for the $u$ and $d$ quark distribution by using a fitted set of parameters. This will give a deeper understanding of both the spatial and transverse distribution of the quark densities.

\section{Nucleon charge densities}

The nucleon form factors are defined in terms of the electromagnetic current operator $J^{\mu}(x)$ as $[9,20]$

$$
\left\langle p^{\prime}, \lambda^{\prime}\left|J^{\mu}(0)\right| p, \lambda\right\rangle=\bar{u}\left(p^{\prime}, \lambda^{\prime}\right)\left(\gamma^{\mu} F_{1}\left(q^{2}\right)+i \frac{\sigma^{\mu \nu}}{2 M} q_{\alpha} F_{2}\left(q^{2}\right)\right) u(p, \lambda),
$$


where $q_{v}=p_{v}^{\prime}-p_{v}$ is the momentum transfer and in the present case it is taken as space-like $\left(q^{+}=0\right)$ leading to $-q^{2}=Q^{2}$. The $\sigma^{\mu v}$ is the spin operator, $\gamma^{\mu}$ denote the Dirac matrices and $u(p, \lambda)$ for the Dirac spinor with mass $M$. The initial (final) momentum and helicity of the nucleon are taken as $p, \lambda\left(p^{\prime}, \lambda^{\prime}\right)$ respectively. The Sachs form factors [17] can be defined in terms of the Dirac and Pauli form factors as follows

$$
G_{E}\left(q^{2}\right)=F_{1}\left(q^{2}\right)-\frac{q^{2}}{4 M^{2}} F_{2}\left(q^{2}\right), G_{M}\left(q^{2}\right)=F_{1}\left(q^{2}\right)+F_{2}\left(q^{2}\right) .
$$

Transverse charge density for a unpolarized nucleon $\rho\left(b_{\perp}\right)$ gives the charge density of the nucleon at a transverse position $b_{\perp}$ for any value of the longitudinal momentum or position. It can be obtained by integrating the ipdpdfs over the longitudinal momentum fraction $x$ and can be expressed in terms of the Dirac form factor [7] and further in terms of $G_{E}$ and $G_{M}$ the transverse charge density for unpolarized nucleon can be expressed as

$$
\rho\left(b_{\perp}\right)=\int_{0}^{\infty} \frac{q d q}{2 \pi} J_{0}\left(q b_{\perp}\right) \frac{G_{E}\left(q^{2}\right)+\tau G_{M}\left(q^{2}\right)}{1+\tau},
$$

where $J_{0}$ is a cylindrical Bessel function and $\tau=\frac{q^{2}}{4 M^{2}}$.

The Dirac and Pauli form factors can be expressed as overlaps of the light front wave functions (LFWFs) for the two-particle Fock state. We consider here a spin- $\frac{1}{2}$ system as a composite of spin- $\frac{1}{2}$ fermion and spin-1 vector boson. The details of the model have been presented in Ref. [21, 22, 23].

The Dirac and Pauli form factor defined in terms of the spin non-flip and spin flip matrix elements of the $J^{+}$current are expressed in Ref. [22]. The transverse charge density from Eq. (2.3) in terms of Sachs form factors can now be written as

$$
\begin{aligned}
\rho\left(b_{\perp}\right)= & \int_{0}^{\infty} \frac{d Q Q}{2 \pi} J_{0}\left(Q b_{\perp}\right) \int_{0}^{1} d x \frac{(1+\tau)\left(2 M^{4}\left(1+x^{2}\right)(1-x) I_{1}+\frac{2(M x-m)^{2}(1-x)^{3} I_{2}}{x^{2}}\right)}{1+\tau}+ \\
& \frac{Q^{2} M^{3}(M x-m) x(1-x)^{3} I_{2}-4 \tau M^{5}(M x-m) x(1-x)^{3} I_{2}}{1+\tau}
\end{aligned}
$$

where

$$
\begin{gathered}
I_{1}=\pi \int_{0}^{1} \frac{\alpha(1-\alpha)}{2 D^{2}} d \alpha, I_{2}=\pi \int_{0}^{1} \frac{\alpha(1-\alpha)}{D^{3}} d \alpha, \\
D=\alpha(1-\alpha)(1-x)^{2} q_{\perp}^{2}-M^{2} x(1-x)+m^{2}(1-x)+\lambda^{2} x .
\end{gathered}
$$

In Fig. 1 we have presented the result for nucleon charge density as a function of impact parameter. For numerical calculations we have taken the value of masses of external fermion, internal fermion and internal boson as $M=m=0.5 \mathrm{MeV}$ and $\lambda=0.02 \mathrm{MeV}$ respectively after imposing the condition $M<m+\lambda$ to prevent nucleon decay [24]. From the figure, we find that the charge density is peaked near $b_{\perp}=0$ and decreases as the value of $b_{\perp}$ increases which implies a maximum distribution near the center of momentum.

\section{Charge density in transverse coordinate space}

The LFWFs in transverse coordinate space $\tilde{\psi}\left(x, \vec{r}_{\perp}\right)$ can be obtained by Fourier transforming $\psi\left(x, \vec{k}_{\perp}\right)$ in the momentum space

$$
\tilde{\psi}\left(x, \vec{r}_{\perp}\right)=\int \frac{d^{2} k_{\perp}}{(2 \pi)^{2}} e^{i \vec{k}_{\perp} \cdot \vec{r}_{\perp}} \psi\left(x, \vec{k}_{\perp}\right),
$$




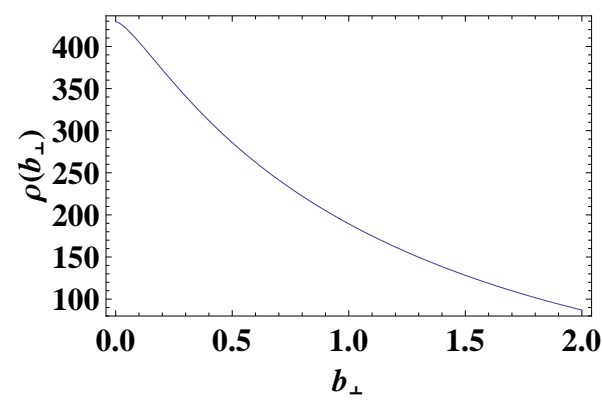

Figure 1: Nucleon charge density (in $\mathrm{fm}^{-2}$ ) as a function of $b_{\perp}$ (in $\mathrm{fm}$ ).

where $\vec{r}_{\perp}$ is the transverse coordinate. The LFWFs for the spin up state in the transverse coordinate space can now be expressed as

$$
\begin{aligned}
& \tilde{\psi}_{+\frac{1}{2}+1}^{\uparrow}\left(x, \vec{r}_{\perp}\right)=i \frac{e}{8 \pi} \sqrt{2} M^{2}(1-x)^{\frac{3}{2}} r_{\perp} K_{0}\left(\sqrt{D} r_{\perp}\right), \\
& \tilde{\psi}_{+\frac{1}{2}-1}^{\uparrow}\left(x, \vec{r}_{\perp}\right)=-i \frac{e}{8 \pi} \sqrt{2} M^{2} x \sqrt{1-x} r_{\perp} K_{0}\left(\sqrt{D} r_{\perp}\right), \\
& \tilde{\psi}_{-\frac{1}{2}+1}^{\uparrow}\left(x, \vec{r}_{\perp}\right)=-\frac{e}{4 \pi} \sqrt{2} M^{2}(M x-m)(1-x)^{\frac{3}{2}} \frac{1}{\sqrt{D}} r_{\perp} K_{1}\left(\sqrt{D} r_{\perp}\right),
\end{aligned}
$$

whereas for spin down state we have

$$
\begin{aligned}
& \tilde{\psi}_{+\frac{1}{2}-1}^{\downarrow}\left(x, \vec{r}_{\perp}\right)=-\sqrt{2} \frac{e}{4 \pi} M^{2}(M x-m)(1-x)^{\frac{3}{2}} \frac{r_{\perp}}{\sqrt{D}} K_{1}\left(\sqrt{D} r_{\perp}\right), \\
& \tilde{\psi}_{-\frac{1}{2}+1}^{\downarrow}\left(x, \vec{r}_{\perp}\right)=i \frac{e}{8 \pi} \sqrt{2} M^{2} x(1-x)^{\frac{1}{2}} r_{\perp} K_{0}\left(\sqrt{D} r_{\perp}\right), \\
& \tilde{\psi}_{-\frac{1}{2}-1}^{\downarrow}\left(x, \vec{r}_{\perp}\right)=-i \frac{e}{8 \pi} \sqrt{2} M^{2} r_{\perp}(1-x)^{\frac{1}{2}} K_{0}\left(\sqrt{D} r_{\perp}\right),
\end{aligned}
$$

where $K$ is the Bessel function of second kind.

In order to compare the nucleon charge density in impact parameter space $\left(\rho\left(b_{\perp}\right)\right)$ with the density distribution in the transverse coordinate space, we first define the density distribution in the transverse coordinate space as

$$
\begin{aligned}
P\left(\vec{r}_{\perp}\right)= & \int d x \frac{e^{2}}{16 \pi^{2}}\left[\frac{1}{2} M^{4}(1-x) r_{\perp}^{2}\left(1+x^{2}\right) K_{0}^{2}\left(\sqrt{D} r_{\perp}\right)+2(M x-m)^{2} M^{4}\right. \\
& \left.\times \frac{(1-x)^{3}}{D} r_{\perp}^{2} K_{1}^{2}\left(\sqrt{D} r_{\perp}\right)\right] .
\end{aligned}
$$

A precise relation between the nucleon density distribution $P\left(r_{\perp}\right)$ and charge distribution $\rho\left(b_{\perp}\right)$ can be obtained using elementary convolution theorems and Fourier transforms is given as

$$
\rho\left(x, \vec{b}_{\perp}\right)=\frac{1}{(1-x)^{2}} P\left(x, \frac{\vec{b}_{\perp}}{-1+x}\right) .
$$

In order to obtain the explicit contributions of the $u$ and $d$ quarks in the nucleon density distribution $P\left(r_{\perp}\right)$ and charge distribution $\rho\left(b_{\perp}\right)$, we have followed the formalism used in Ref. [7] where the 

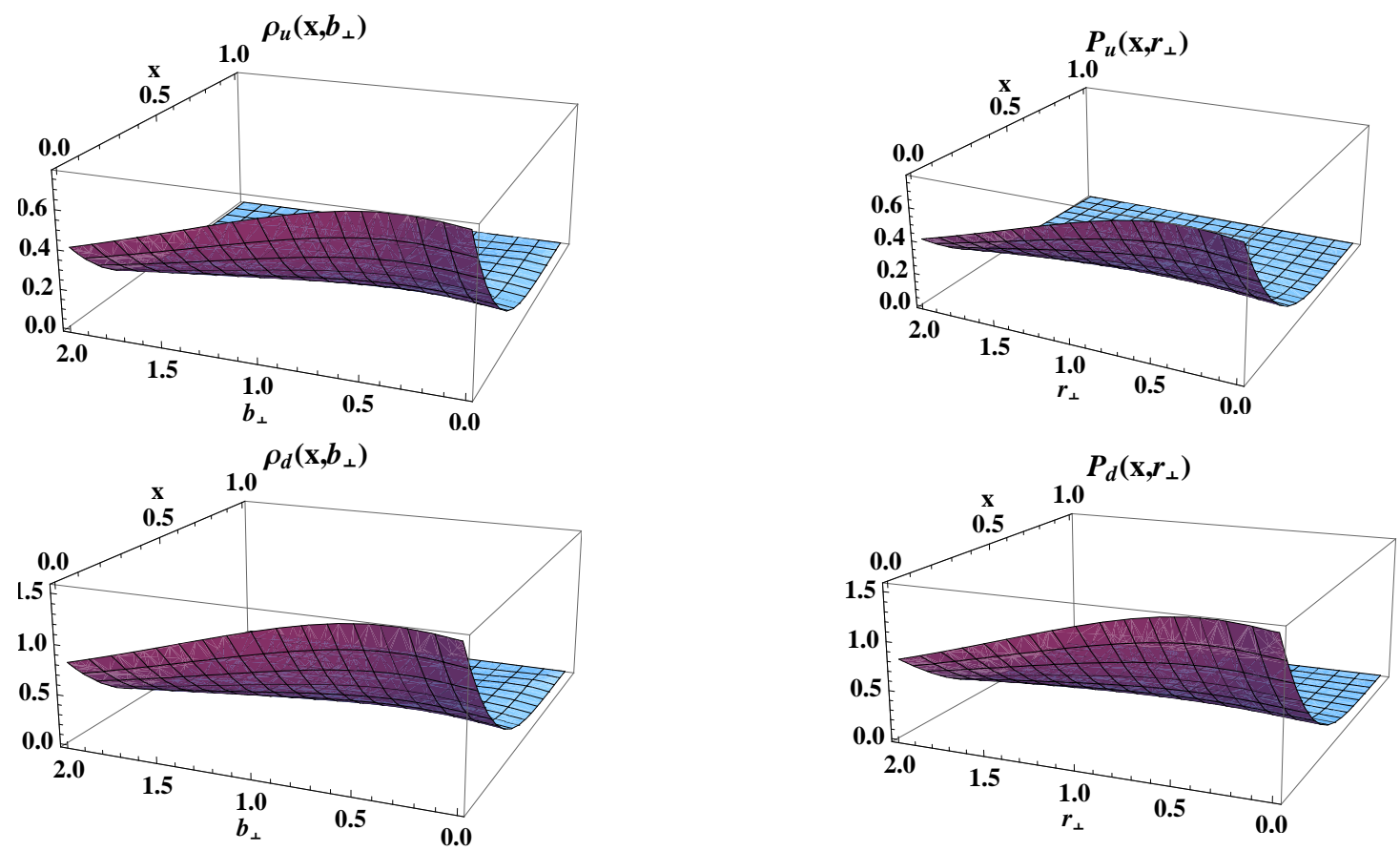

Figure 2: Results for $\rho_{u(d)}\left(x, b_{\perp}\right)$ (in $f m^{-2}$ ) and $P_{u(d)}\left(x, r_{\perp}\right)$ (in $f m^{-2}$ ) for the up (down) quark in $b_{\perp}$ (in $f m$ ) and $r_{\perp}$ (in $f m$ ) space respectively.

isospin symmetry $P_{u}=P_{p}+\frac{P_{n}}{2}, P_{d}=P_{p}+2 P_{n}$ has been used with similar type of relations for charge density in the impact parameter space. A fitting can be carried out with the experimental data of the Dirac form factors of the nucleon to obtain the fitted values of the masses of the internal fermions and bosons. For the present work we have used the same set of fitted values of $m, \lambda_{u}$ and $\lambda_{d}$ as in Ref. [7, 18, 25].

In Fig. 2, we have presented the result for density distribution and charge distribution for $u$ and $d$ quarks respectively. These plots give the complete spatial information about the nucleon. It is clear from the plots that charge and density distributions increase with the increasing value of the impact parameter $b_{\perp}$ and the transverse coordinate $r_{\perp}$ respectively, reach a maxima and then start decreasing. The peak for the charge distribution in impact parameter space is around $b_{\perp} \sim 0.4 \mathrm{fm}$ whereas the peak for the density distribution in transverse coordinate space $r_{\perp} \sim 0.6 \mathrm{fm}$ for both the $u$ and $d$ quarks. The magnitudes of $\rho_{u}\left(b_{\perp}\right)$ and $P_{u}\left(r_{\perp}\right)$ are however different from $\rho_{d}\left(b_{\perp}\right)$ and $P_{d}\left(r_{\perp}\right)$. A closer look at the figure reveals that the density distribution in transverse coordinate space falls off slowly as compared to the charge distribution in impact parameter space. This is due to the factor $\frac{b_{\perp}}{-1+x}$ appearing in Eq. (3.5).

\section{Conclusions}

In the present work, we have considered the light-cone Fock state representation of the composite system consisting of external and internal fermion state with respective masses $M$ and $m$. The mass of the vector constituent is taken to be $\lambda$. The electromagnetic Dirac and Pauli form factors as well as the Sachs form factors $\left(G_{E}\left(Q^{2}\right)\right)$ and $\left(G_{M}\left(Q^{2}\right)\right)$ have been calculated from the overlap 
of LFWFs. The nucleon charge density has been obtained for the case of unpolarized nucleon in transverse coordinate space as well as in the impact parameter space. Further, a relation has been developed between the charge distribution of the quarks inside nucleon in the transverse coordinate space as well as in the impact parameter space particularly for the $u$ and $d$ quark distribution by using a fitted set of parameters.

\section{References}

[1] M. K. Jones et al., Phys. Rev. Lett. 84 , 1398 (2000).

[2] O. Gayou et al., Phys. Rev. Lett. 88, 092301 (2002).

[3] V. P. Druzhinin, arXiv:1311.7517.

[4] A. J. R. Puckett et al., Phys. Rev. Lett. 104, 242301 (2010).

[5] J. C. Bernauer et al. (A1 Collaboration), Phys. Rev. Lett. 105, 242001 (2010).

[6] G. Ron et al. (Jefferson Lab Hall A Collaboration), Phys. Rev. C 84, 055204 (2011).

[7] D. S. Hwang, D. S. Kim and J. Kim, Phy. Lett. B 669, 345 (2008).

[8] O. V. Selyugin, O. V. Teryaev, Phys. Rev. D 79, 033003 (2009).

[9] M. Diehl, Phys. Rept. 388, 41 (2003).

[10] X. Ji, Annu. Rev. Nucl. Part. Sci 54, 413 (2004).

[11] M. Diehl, Eur. Phys. C 25, 223 (2002).

[12] M. Burkardt, Phys. Rev. D 62, 071503 (2000).

[13] R. Manohar, A. Mukherjee and D. Chakrabarti, Phys. Rev. D 83, 014004 (2011).

[14] D. E. Soper, Phys. Rev. D 15, 1141 (1977).

[15] G. A. Miller, J. Arrington, Phys. Rev. C, 78, 032201 (2008).

[16] S. Venkat, J. Arrington, G. A. Miller and X. Zhan, Phys. Rev. C 83, 015203 (2011).

[17] M. Diehl, Phys. Rev. 126, 2256 (1962).

[18] J. J. Kelly, Phys. Rev. C 70, 068202 (2004).

[19] Z. Abidin, C. E. Carlson, Phys. Rev. D 78, 071502 (2008).

[20] M. Guidal, M. V. Polyakov, A. V. Radyushkin and M. Vanderhaeghen, Phys. Rev. D 72, 054013 (2005).

[21] G. P. Lepage, S. J. Brodsky, Phys. Rev. D 22, 2157 (1980).

[22] S. J. Brodsky, D. S. Hwang, B. Q. Ma and I. Schmidt, Nucl. Phys. B 593, 311 (2001).

[23] D. Chakrabarti, A. Mukherjee, Phys. Rev. D 72, 034013 (2005).

[24] S. J. Brodsky, D. Chakrabarti, A. Harindranath, A. Mukherjee and J. P. Vary, Phys. Rev. D 75, 014003 (2007).

[25] J. J. Kelly, Phys. Rev. C 66, 065203 (2002). 